\title{
Active DC Bus Signaling Control Method for Coordinating Multiple Energy Storage Devices in DC Microgrid
}

\author{
Fulong Li, Zhengyu Lin \\ PEMPS research group \\ Aston University \\ Birmingham, B4 7ET, UK \\ lif12@aston.ac.uk
}

\author{
Zhongnan Qian, Jiande Wu \\ College of Electrical Engineering \\ Zhejiang University \\ Hangzhou, 310027, China
}

\begin{abstract}
Management of multiple energy storage devices in a DC microgrid is a challenge. Conventional method, such as droop control, cannot ensure accurate current sharing in coordinating multiple battery banks, which limits the DC microgrid system performance. This paper proposed an active DC bus signaling (ADBS) method to coordinate multiple battery banks in a DC microgrid. It has the advantages of accurate current sharing. Using the proposed ADBS method, the master controller can collect State of charge (SoC) of each slave battery bank by actively varying the DC bus voltage levels and monitoring the current change. The master module then sets a working voltage level after decision-making. The proposed method was experimentally evaluated, and the experimental results show that all the possible working voltage levels can be reached and the accuracy of current sharing is guaranteed.
\end{abstract}

Keywords-DC microgrid; control strategy; DC bus signaling; multiple energy storage devices

\section{INTRODUCTION}

Microgrids are a small-scale power subsystem that include distributed generation, energy storage, local load, system control and coordination. Distributed generations can be connected through DC links or AC links. According to the history perspective, $\mathrm{AC}$ has been the standard choice. However, with the development of power electronics technologies, DC power supply systems are gaining increasing interest in past several years. Compared with conventional AC systems, DC systems have advantages of higher reliability and efficiency, simpler control and natural interface to increased numbers of DC renewable generations, energy storage and electronic loads.

Battery is the most popular energy storage device in DC microgrids. Usually, a DC microgrid includes only one battery bank. However, as the popularity of electrical vehicles and reuse of second-life batteries, multiple battery banks can be seen in DC microgrids. This situation poses a new challenge for coordinating the multiple battery banks in a DC microgrid.

Droop control is one of the optional method to coordinate the multiple battery banks. However, the performance is limited by its poor current sharing [1]. Even if there are many improved adaptive droop control methods [2-4] proposed to increase the current sharing accuracy, this method still cannot acquire the state information of each battery bank, which means this method lacks the function of information exchange. DC bus signaling (DBS) method compensates this drawback. DBS method [5] can ensure the system work under different modes through setting different voltage thresholds. DBS method was developed from voltage level signaling [6], which is, however, limited by its expandability. Adding droop loop between the two voltage levels makes it become DBS method. Therefore, DBS method still has the same drawback in current sharing as the droop control method. There is also another DBS method proposed in [7]. This DBS method is organized as master-slave (M-S) mode, which means that picking one power source as the master module among all power sources and the rest as slave modules. The master module works at voltage controlled mode, mainly responsible for the DC bus voltage variation, while salve modules work at current controlled mode, ensuring accurate current input or output. This method can ensure the current sharing accurate, but it cannot acquire the information from other distributed generations.

Managing all battery banks in centralized manner can improve the current sharing accuracy. However, it adds the cost of additional communication circuits, which is not suitable for small scale DC microgrids in remote area. Apart from that, centralized control also has the drawback of single point of failure.

Using the proposed active DC bus signaling (ADBS) method, Master controller can actively collect the state of charge $(\mathrm{SoC})$ information of each battery bank. The control mode is based on master-slave (M-S) mode. The master module actively sets the DC bus voltage at different levels to switch ON/OFF slave modules. Each slave module has its own working voltage levels. When one of the salve module is switched on, it will then release or absorb a current signal that can be sensed by the master module so that SoC information can be attained. This process will be carried on periodically.

The rest of this paper is organized as follows: Section II gives the illustration of system configuration and explains the control principle. Section III shows the experimental setup and results. Finally, the concluding remarks and future work can be found in Section IV. 


\section{EXPLAINATION OF PROPOSED CONTROL METHOD}

\section{A. Configuration of system}

A typical configuration of DC microgrid with multiple energy storage devices can be seen in Fig.1. It contains three main parts, namely, energy storage devices, power generations and power consumptions.

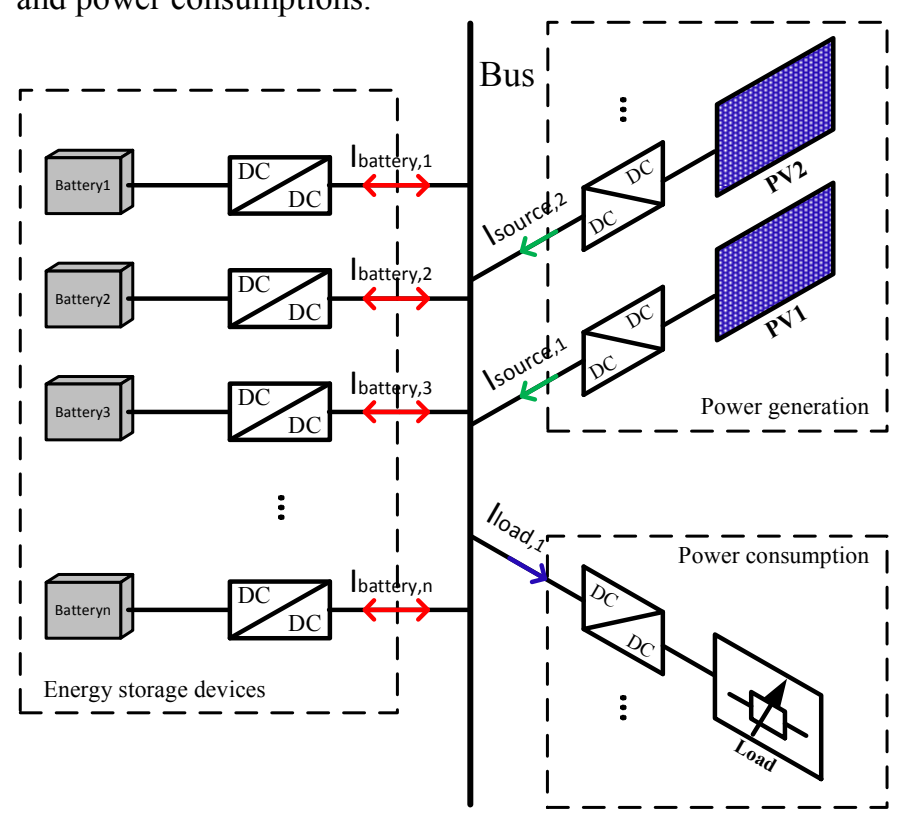

Fig. 1. Configuration of a typical DC mcirogrid

The power generations include PV, wind turbine and fuel cell, which only provide power. The green arrow in Fig.1 shows the power flow. In contrast, the load only consume power from the microgrid and the blue arrow shows the power flow. Battery banks can absorb or release power, which is labeled with red arrow.

\section{B. Explaination of voltage arrangement}

According to the Fig.1, if denoting all the input current (green arrow) of distributed sources (e.g. PV) as

$$
I_{s 1}, I_{s 2}, \cdots, I_{s, l}
$$

all the battery bi-directional current (red arrow) as

$$
I_{b 1}, I_{b 2}, \cdots, I_{b, m}
$$

and all load current (blue arrow) as

$$
I_{l 1}, I_{l 2}, \cdots, I_{l, n}
$$

According to the Kirchhoff current law,

$$
\sum_{i=1}^{l} I_{s, i} \pm \sum_{j=1}^{m} I_{b, j}-\sum_{k=1}^{n} I_{l, n}=0
$$

Where if the battery work at discharging mode, it will be positive.
If choose one battery bank as the master module, the master current can be written as

$$
I_{b 1}=\sum_{i=1}^{l} I_{s, i} \pm \sum_{j=2}^{m} I_{b, j}-\sum_{k=1}^{n} I_{l, n}
$$

The proposed method targets at one PV module, three battery banks. The battery1 was chosen as the master module, therefore, the equation (5) can be shorten as

$$
\mp_{b 1}=I_{P V}-I_{l} \pm I_{b 2} \pm I_{b 3}
$$

According to the equation (6), if the PV works at maximum power point and the load is kept constant a certain period. The current variation in slave modules can be reflected by the master module.

In order to arrange DC bus voltage levels for each salve module, seven voltage levels are set in this study. Fig. 2 shows the DC bus voltage arrangement for the proposed ADBS method.

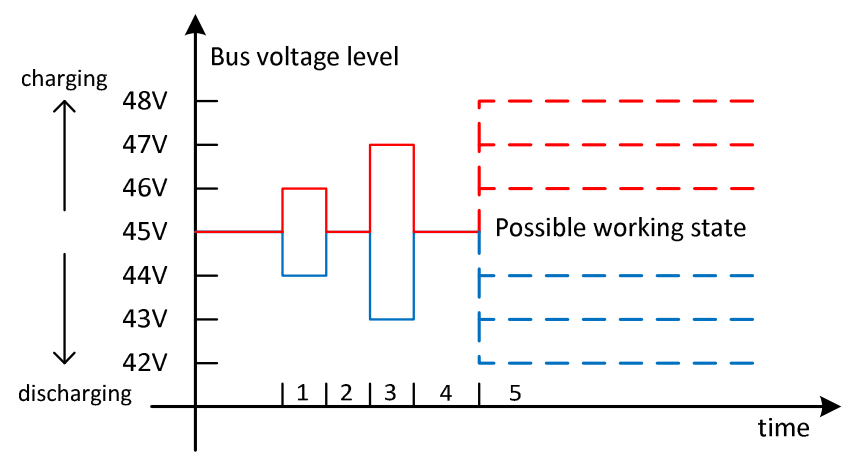

Fig. 2. DC bus voltage arrangement for the ADBS method

$45 \mathrm{~V}$ was set as initial DC bus voltage. The DC bus voltages above $45 \mathrm{~V}$ were set as charging mode while below $45 \mathrm{~V}$ set as discharging mode. $46 \mathrm{~V}$ and $44 \mathrm{~V}$ are set to wake up slave1, and $47 \mathrm{~V}$ and $43 \mathrm{~V}$ are set to wake up salve $2.48 \mathrm{~V}$ and 42 are set to wake up both but different working mode when the discharging or charging current in master module is larger than 4A. The reason of setting two working voltage levels is that each slave battery has two possible working modes. Different voltage levels make them easy to choose work at charging or discharging mode. It is also achievable to set one voltage level to realize both working modes.

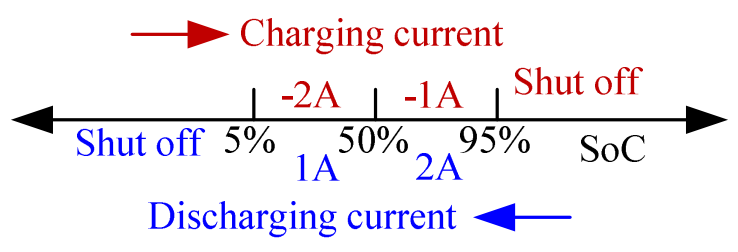

Fig. 3. Reference current setting through SoC 
In slave module, the discharging and charging current is defined according to the state of charge (SoC). Fig.3 gives the relationship between reference current and SoC.

When it is in discharging mode, higher SoC will release more power to the microgrid. In contrast, when it is in charging mode, lower SoC will absorb more power from the microgrid. The relationship can also be modified according to real needs.

\section{Analysis of control methods}

The proposed ADBS method is M-S based control method, which means the master module works under voltage control mode and the salve module works under current control mode.

The control block diagram of the master module is shown in Fig.4. The master module is responsible for actively setting different DC bus voltage levels to inform salve modules. The reference voltage is decided by the current variation in the master module.

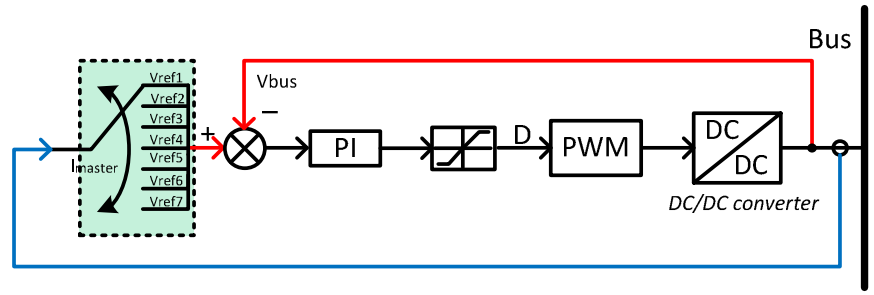

Fig. 4. Control block diagram of the master module

The control block diagram of slave modules is current control mode, which is shown in Fig.5. The current reference value is decided by the SoC. One benefit of this is that the slave module has the ability to automatically adjust the charging or discharging current by itself.

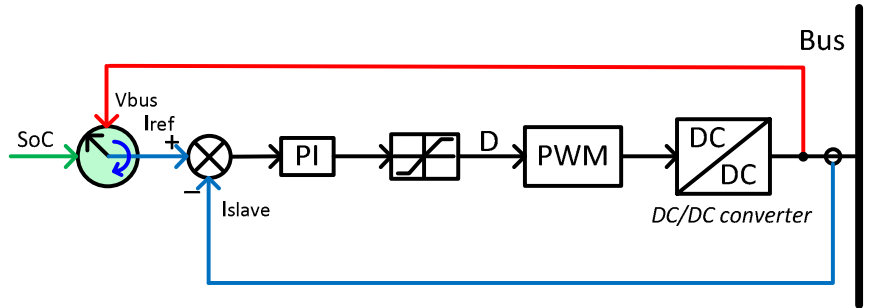

Fig. 5. Control block diagram of the salve module

The control block of the PV module is shown in Fig.6. The PV module works at maximum power point, which was implemented by Perturb and Observe (P\&O) MPPT algorithm embedded in PV converter module.

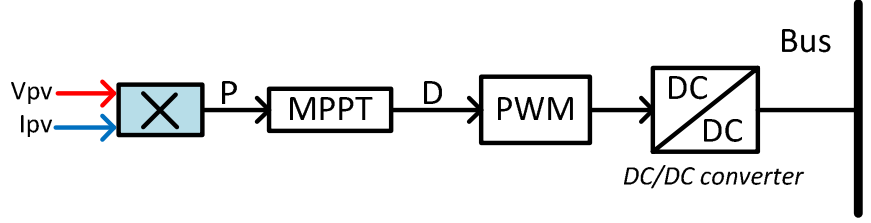

Fig. 6. Control block diagram of the PV module

\section{EXPERIMETNAL VALIDATIOIN}

A DC microgrid experimental test system was built to validate the proposed ADBS method. Fig.7 shows the experimental setup, which includes three battery banks, a PV emulator and a variable DC load.

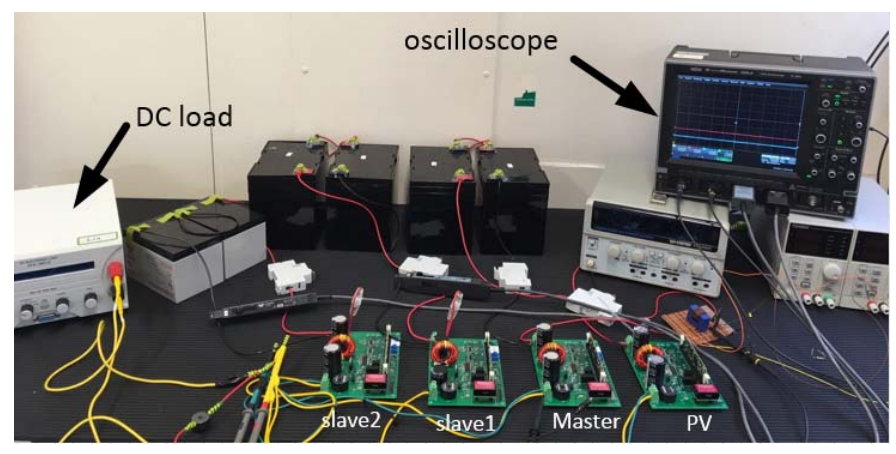

Fig. 7. experimental setup

Table I lists the equipment in the experimental setup.

TABLE I. EQUIPMENT LIST

\begin{tabular}{|c|c|c|}
\hline \multirow{2}{*}{} & \multicolumn{2}{|c|}{ Typecifications } \\
\cline { 2 - 3 } PV emulator & $62050 \mathrm{H}-600 \mathrm{~S}$ & Number \\
\hline Battery & YPC33-12 & 4 \\
\hline Battery & NP12-12 & 2 \\
\hline DC load & EA-EL 3400-25 & 1 \\
\hline Boost converter & 200W & 4 \\
\hline
\end{tabular}

The experiment validation contains two parts: discharging and charging process respectively.

\section{A. Discharging experimental results}

This situation takes place when the power generations is zero or low. For example, the PV panels are disconnected or work at cloudy or rainy days.

Fig. 8 shows the experimental result. After setting the DC bus voltage at $44 \mathrm{~V}$ and $43 \mathrm{~V}$ to obtain the SoC information of slave batteries, the final working DC bus voltage level is set as $44 \mathrm{~V}$. The initial current in master module is $2.6 \mathrm{~A}$, which is lower than the total discharging current of both slave batteries (4A). Therefore, the master module will pick up one slave to share the discharging current. Firstly, the master module actively set the DC bus voltage level at $44 \mathrm{~V}$ to collect the SoC of slave1 module, and then set it as $43 \mathrm{~V}$ to collect slave2 module. In Fig.8, it shows that slave1 module discharges 1A current when the DC bus voltage was set at $44 \mathrm{~V}$, while slave 2 module discharges $0 \mathrm{~A}$ current when the DC bus voltage was set at $43 \mathrm{~V}$. It means that slave 1 module has more capacity than slave1. Therefore, the final working voltage is set at $44 \mathrm{~V}$ after decision-making, where master and slavel module share the discharging current.

One thing needs to be mentioned is that the area A1 in Fig.7, it shows a current drop when the bus voltage is set to 
$43 \mathrm{~V}$. This is because of the constant resistance load. When voltage drops, load current drops.

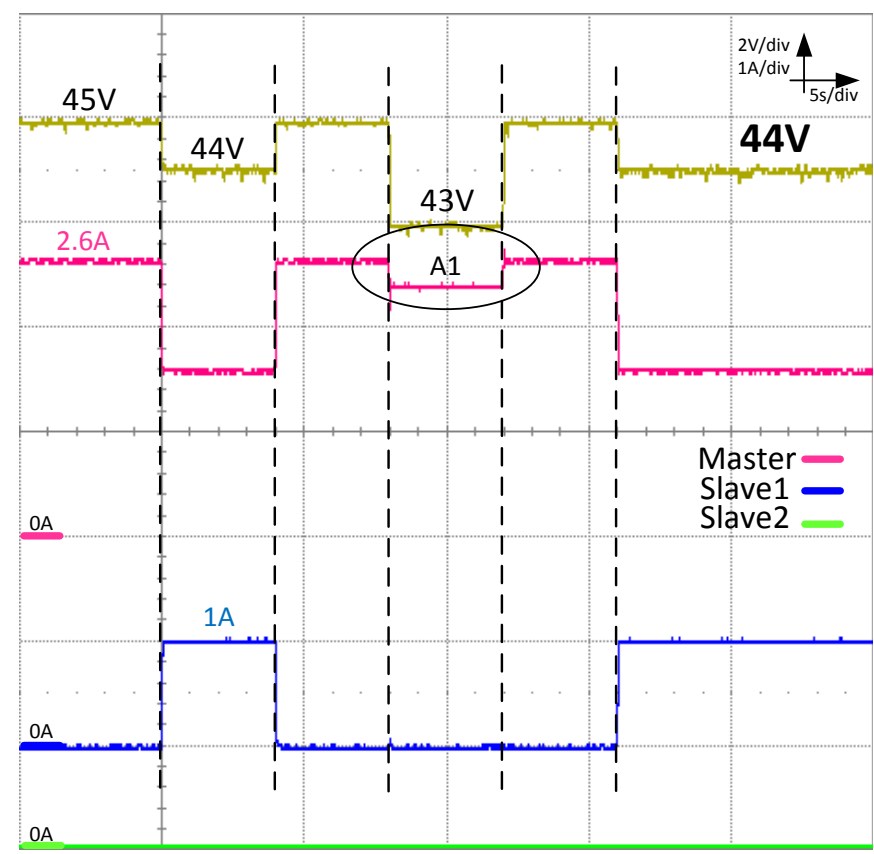

Fig. 8. Final working DC bus voltage level at $44 \mathrm{~V}$ after decision-making

Fig.9 shows the experiment result with final working DC bus voltage level of $43 \mathrm{~V}$. The experiment condition is same as the previous one. The difference is that the slave 2 module has more capacity than the slave1 module. Therefore, the final working DC bus voltage level is set as $43 \mathrm{~V}$, where master and salve2 share the discharge current.

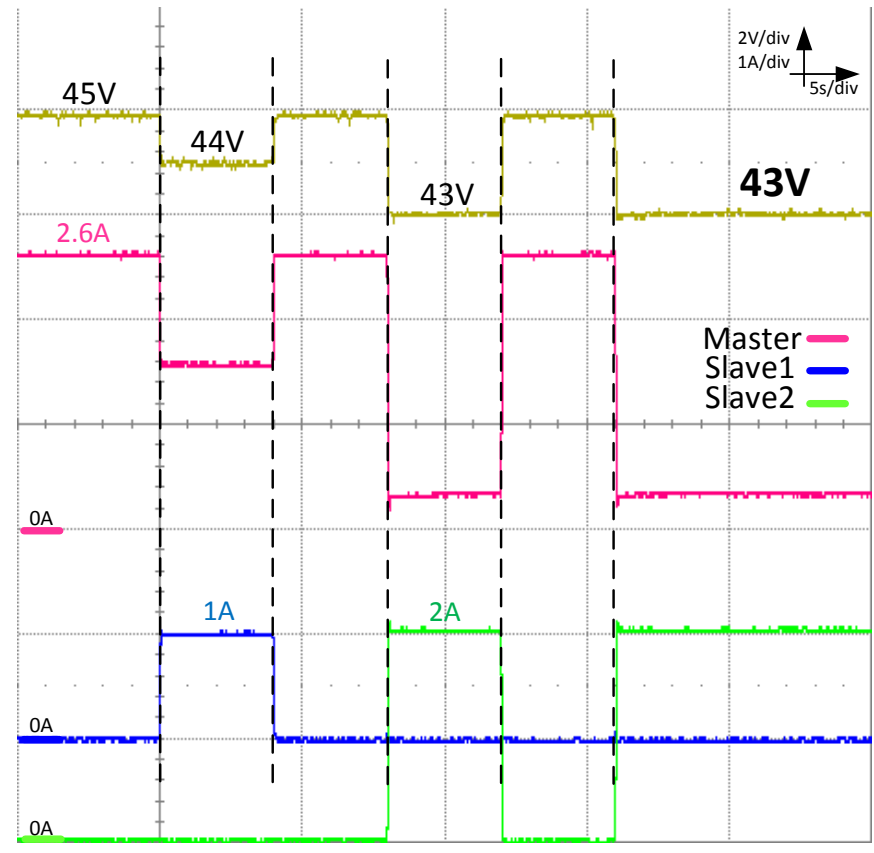

Fig. 9. Final working DC bus voltage level at $43 \mathrm{~V}$ after decision-making
When the initial discharging current in master module is larger than 4A, for example, is 4.7A as shown in Fig.10, the master module will set the voltage at $42 \mathrm{~V}$ to force both the slave1 and slave2 to discharge unless very low SoC. Each slave module also has self-protection algorithm, which is that they will shut off themselves at low SoC until they receive the charging voltage levels.

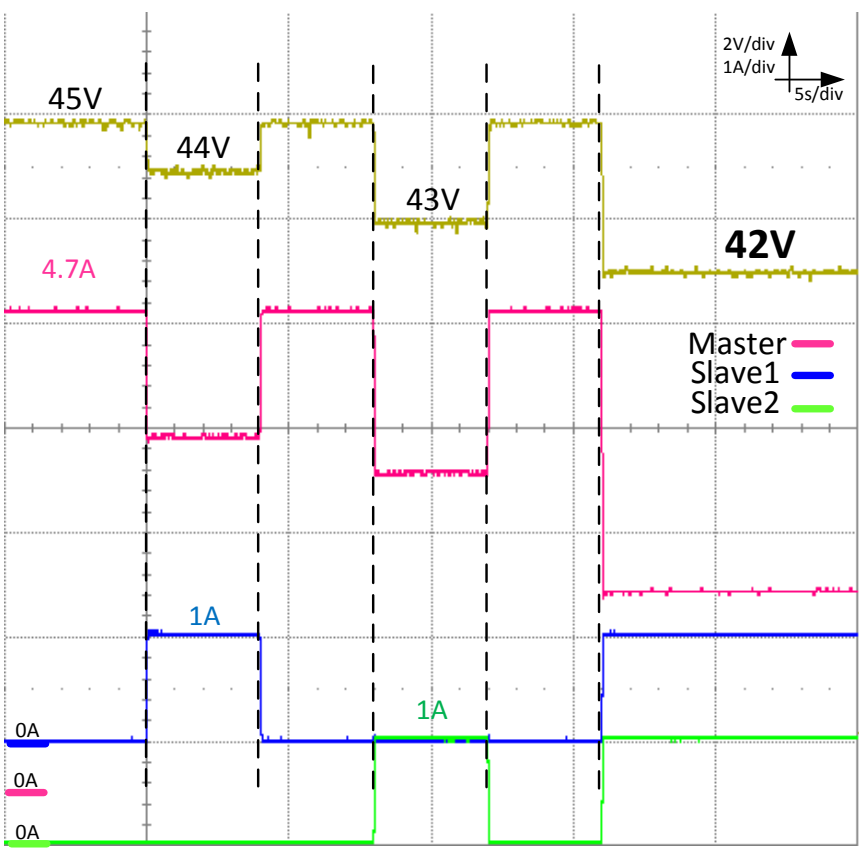

Fig. 10. Final working DC bus voltage level at $42 \mathrm{~V}$ after decision-making

\section{B. Charing Experimental Analysis}

If the PV works at sunny days and the system has no heavy loads, this means that it has additional power to charge the battery. In this situation, the system will work at higher DC bus voltage levels to charge the batteries.

Fig.11 shows experimental result with the final working $\mathrm{DC}$ bus voltage level of $46 \mathrm{~V}$. PV is charging the master battery module, and the initial charging current is $2.8 \mathrm{~A}$, which is lower than the total charging current of both slave battery modules (4A). Firstly, the master module actively set the DC bus voltage level at $46 \mathrm{~V}$ to collect the SoC of slavel module, and then $47 \mathrm{~V}$ to collect slave 2 module. In Fig.11, it can be seen that slave1 module charges with $1 \mathrm{~A}$ when the bus voltage was set at $46 \mathrm{~V}$ while slave 2 module charges with $0 \mathrm{~A}$ when the bus voltage was set at $47 \mathrm{~V}$, which means that slave1 module has lower capacity than slave 2 module. After the decision-making, the final working DC bus voltage levels will be set at $46 \mathrm{~V}$, where the slavel module and master module share the charging current. 


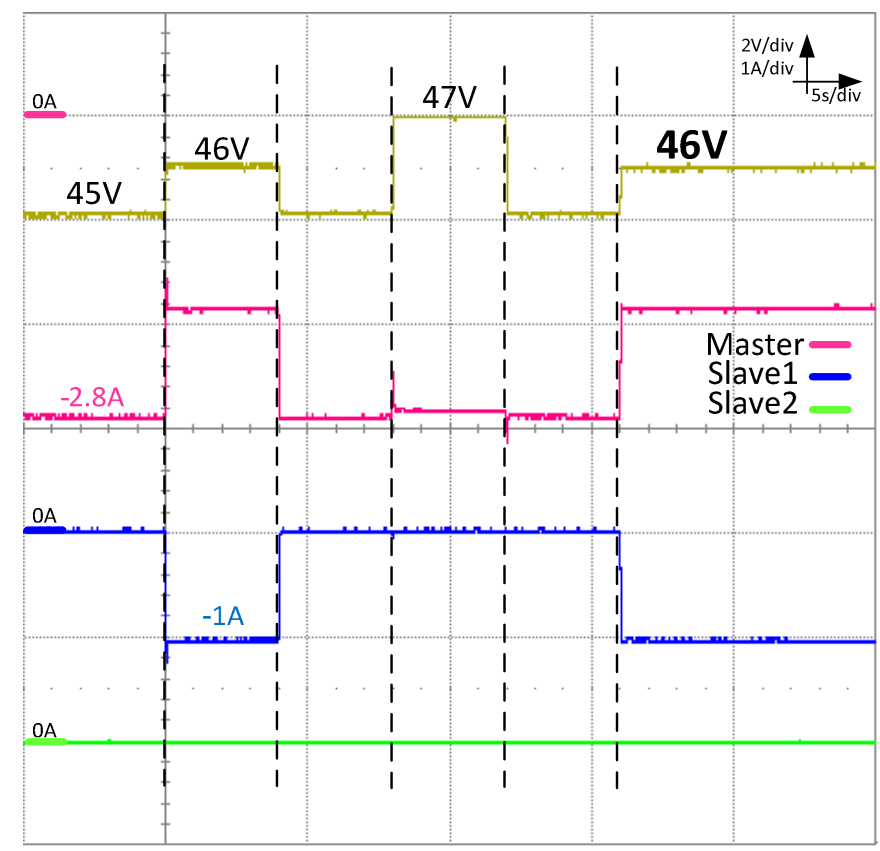

Fig. 11. Final working DC bus voltage level at $46 \mathrm{~V}$ after decision-making

Fig. 12 shows the experimental result with the final working DC bus voltage level of $47 \mathrm{~V}$. The experiment condition is same as the previous one. The difference is that the slave2 module presently has lower capacity than the slave1 module. Therefore, the final working DC bus voltage level is set at $47 \mathrm{~V}$, where master and salve2 battery modules are sharing the charging current.

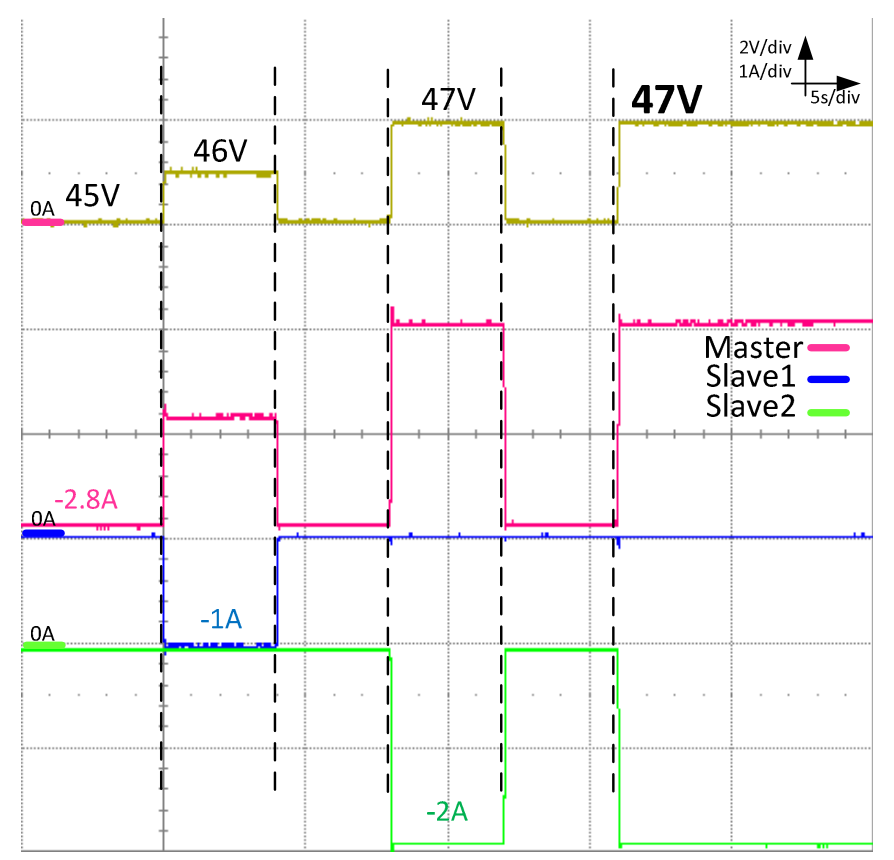

Fig. 12. Final working DC bus voltage level at $47 \mathrm{~V}$ after decision-making

When the initial charging current in master module is larger than $4 \mathrm{~A}$, which is $4.2 \mathrm{~A}$ in Fig.13, the master module will set the voltage at $48 \mathrm{~V}$ to share the charging current with both slaves until they are fully charged. When they are fully charged, they will shut off themselves until they receive the discharging voltage levels.

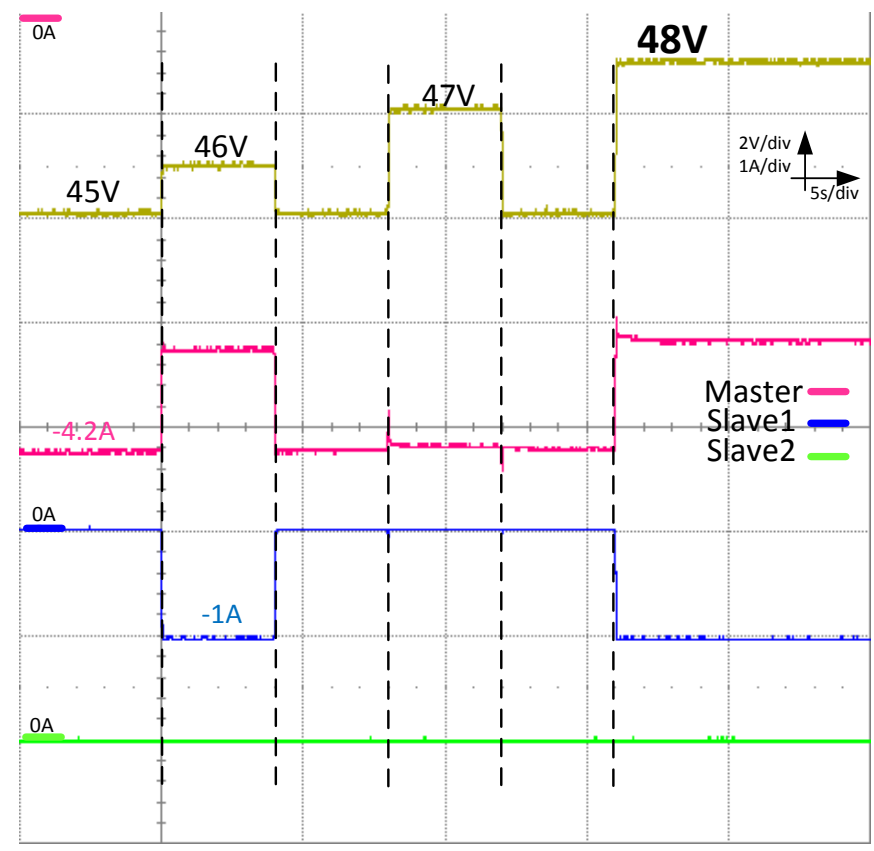

Fig. 13. Final working DC bus voltage level at $48 \mathrm{~V}$ after decision-making

Apart from the above mentioned possible working voltage levels, there are still several issues needed to be discussed. Fig.14 shows a case that slave2 was not connected at first. When it is connected, it will automatically charge or discharge according to bus voltage levels and its own SoC. On the other hands, it cannot affect the decision that has made by the master. It will need to wait next round voltage floating so that its SoC can be acquired by the master module.

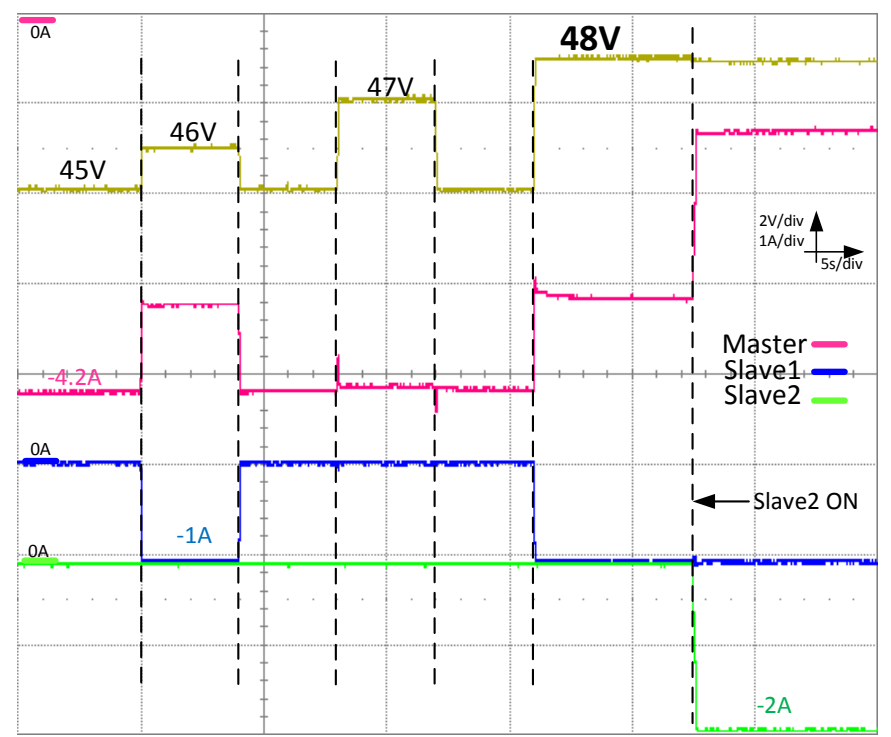

Fig. 14. Final working DC bus voltage level at $48 \mathrm{~V}$ with salve $2 \mathrm{SoC}$ recovery

Fig.15 shows another case when the master module is at low SoC, it will stop sharing power with slaves. The DC bus 
voltage will be back to $45 \mathrm{~V}$. This process will continue until it arrives at the working $\mathrm{SoC}$ value.

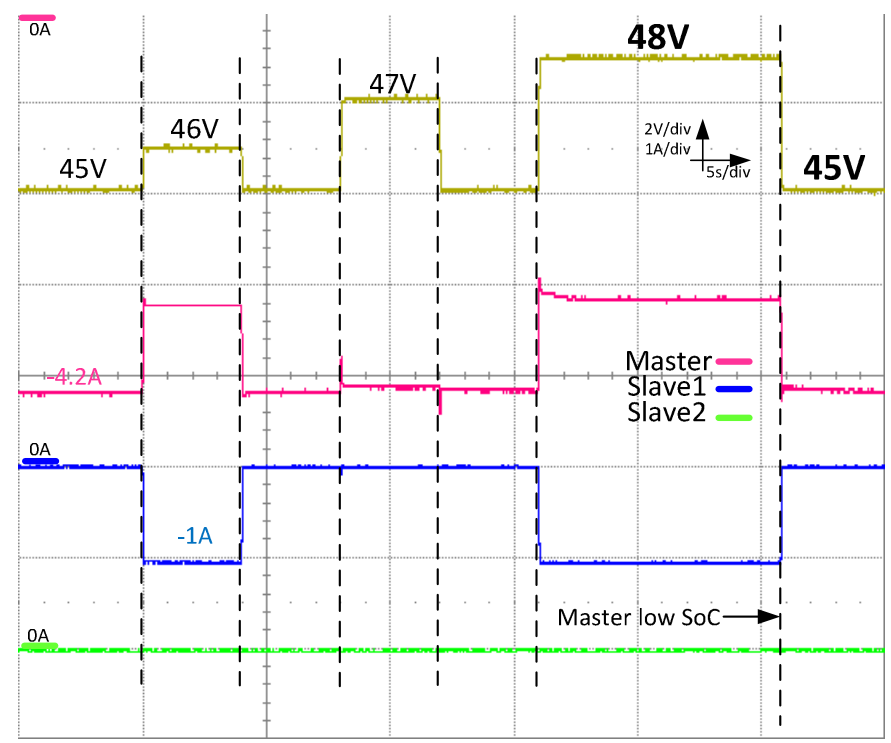

Fig. 15. Final working voltage level at $45 \mathrm{~V}$ for master low SoC

There are still many possible situations in real scenario. However, they do not influence the feasibility of the proposed method and can be solved by improving relative algorithm.

\section{CONCLUSIONS AND FUTURE WORK}

This paper proposed an active DC bus signaling method to coordinate multiple battery banks in a DC microgrid. The proposed method has been experimentally evaluated, and the experimental results show that accurate current sharing can be achieved. The experimental results also show the expandability of the proposed ADBS method.

However, this method has some limitations. Firstly, the number of the battery banks is finite. Adding more battery banks needs more voltage levels to switch ON/OFF slave battery bank modules. The levels of DC bus voltage cannot be too large, which might cause the unstable operation. Secondly, the information exchange is achieved through voltage level, so it is only suitable for the low speed application.

There are several issues needed to be further investigated in future work. Firstly, the step current displayed in the experimental parts is not good for the system operation. Secondly, slave modules cannot inform the master actively, which means when a slave is loaded on, it will need to wait next round voltage floating.

\section{REFERENCES}

[1] X. Lu, J. M. Guerrero, K. Sun and J. C. Vasquez, "An Improved Droop Control Method for DC Microgrids Based on Low Bandwidth Communication With DC Bus Voltage Restoration and Enhanced Current Sharing Accuracy," in IEEE Transactions on Power Electronics, vol. 29, no. 4, pp. 1800-1812, April 2014

[2] Ganesh R, G. Panda and R. Peesapati, "Hardware-in-loop implementation of an adaptive droop control strategy for effective load sharing in DC Microgrid," 2016 IEEE 6th International Conference on Power Systems (ICPS), New Delhi, 2016, pp. 1-6.

[3] S. Anand, B. G. Fernandes and J. Guerrero, "Distributed Control to Ensure Proportional Load Sharing and Improve Voltage Regulation in Low-Voltage DC Microgrids," in IEEE Transactions on Power Electronics, vol. 28, no. 4, pp. 1900-1913, April 2013.K. Elissa, "Title of paper if known," unpublished.

[4] V. Nasirian, A. Davoudi, F. L. Lewis and J. M. Guerrero, "Distributed Adaptive Droop Control for DC Distribution Systems," in IEEE Transactions on Energy Conversion, vol. 29, no. 4, pp. 944-956, Dec. 2014.

[5] J. Schonbergerschonberger, R. Duke and S. D. Round, "DC-Bus Signaling: A Distributed Control Strategy for a Hybrid Renewable Nanogrid," in IEEE Transactions on Industrial Electronics, vol. 53, no. 5, pp. 1453-1460, Oct. 2006.

[6] J. Bryan, R. Duke and S. Round, "Decentralized generator scheduling in a nanogrid using DC bus signaling," IEEE Power Engineering Society General Meeting, 2004., 2004, pp. 977-982 Vol.1.

[7] K. Sun, L. Zhang, Y. Xing and J. M. Guerrero, "A Distributed Control Strategy Based on DC Bus Signaling for Modular Photovoltaic Generation Systems With Battery Energy Storage," in IEEE Transactions on Power Electronics, vol. 26, no. 10, pp. 3032-3045, Oct. 2011. 